\title{
Review Article \\ Game Play Schemas: From Player Analysis to Adaptive Game Mechanics
}

\author{
Craig A. Lindley and Charlotte C. Sennersten \\ Department of Technoculture, Humanities and Planning, Blekinge Technical Institute, Campus Karlshamn, \\ Biblioteksgatan 4, SE-374 35 Karlshamn, Sweden \\ Correspondence should be addressed to Craig A. Lindley, craig.lindley@bth.se
}

Received 31 July 2007; Accepted 19 October 2007

Recommended by Kok Wai Wong

Schema theory provides a foundation for the analysis of game play patterns created by players during their interaction with a game. Schema models derived from the analysis of play provide a rich explanatory framework for the cognitive processes underlying game play, as well as detailed hypotheses for the hierarchical structure of pleasures and rewards motivating players. Game engagement is accounted for as a process of schema selection or development, while immersion is explained in terms of levels of attentional demand in schema execution. However, schemas may not only be used to describe play, but might be used actively as cognitive models within a game engine. Predesigned schema models are knowledge representations constituting anticipated or desired learned cognitive outcomes of play. Automated analysis of player schemas and comparison with predesigned target schemas can provide a foundation for a game engine adapting or tuning game mechanics to achieve specific effects of engagement, immersion, and cognitive skill acquisition by players. Hence, schema models may enhance the play experience as well as provide a foundation for achieving explicitly represented pedagogical or therapeutic functions of games.

Copyright (c) 2008 C. A. Lindley and C. C. Sennersten. This is an open access article distributed under the Creative Commons Attribution License, which permits unrestricted use, distribution, and reproduction in any medium, provided the original work is properly cited.

\section{INTRODUCTION}

Computer game genres, such as role-playing games (RPGs) and first-person shooters (FPSs), imply particular sets of design features supporting expectations that prospective players have about the nature of the play experience that games support, based upon past experiences with other games in the same genres. When a player first encounters a computer game within an unfamiliar genre, they will, if sufficiently motivated, interact with the game and eventually learn sufficient patterns of interaction to make progress within the game, perhaps eventually completing it. Game play is therefore fundamentally a process of players learning, adapting and improving play skills. Since computer games are predominantly played by the use of very generic interaction technologies (e.g., a keyboard and mouse), learning and adaptation in play are, for the most part, processes of developing cognitive skills focused upon the mechanics of a game and its media realization, based upon an existing general skill set for computer use. Keyboard and mouse operations are mapped onto in-game actions in a game world synthesized by the game software. Learning how to play can therefore be divided into three phases: (1) learning interaction mechanics, that is, the basic motor operations required to operate, for example, a keyboard and mouse in a largely unconscious way; (2) learning interaction semantics, that is, the simple associative mappings from keyboard and mouse operations to ingame actions (and meta-game actions, such as setting play options, or loading and saving game states); and (3) learning game play competence, that is, how to select and perform in-game actions in the context of a current game state in a way that supports progress within a game. Interaction semantics represent a basic level of competence in playing a particular game; these mappings are often carried across different games within a genre and even across genres (e.g., using "w," "a," "s," and "d" keys to move a player character forwards, left, backwards and right, resp.). Learning interaction semantics represents a form of game challenge (in addition to those noted by Rollings and Adams [1]), but once the basic mappings have been learned, they become a largely unconscious foundation for ongoing game play. The focus of learning then shifts to the development of game play competence, 
which involves the development of forms of in-game situation awareness and decision making needed to meet the more complex challenges such as those documented by Rollings and Adams [1].

Game play competence involves the ability to (1) decode the audiovisual sensory and perceptual information delivered by the game media (e.g., the computer screen and speakers) into the apprehension of a local situation within the synthesized game world (or game space); (2) evaluate this understanding of the local in-game situation in terms of the overall objectives of play, current goals and tasks, the state of the player character within the game (e.g., capabilities, health, and other statistics), and anticipation of various rewards of playing the game; (3) make decisions about which in-game tactics and action(s) to perform next, based upon the perceived situation and its evaluation; and (4) perform action(s) based upon competence in interaction mechanics and semantics. The details of the cognitive process underlying this repetitive sequence, which could be described as the sense- $>$ model- $>$ evaluate- $>$ plan- $>$ act sequence (essentially the same as the sense->model->plan$>$ act structure used to simulate higher-level action control in robots and agents within artificial intelligence research; see http://www.cgie2006.murdoch.edu.au/game_ai.html for extensive references), are the primary higher-level cognitive learning outcome of learning how to play a particular computer game.

The general usefulness of these different aspects of learning in game play relates to the degree to which the knowledge or skills learned may transfer to other contexts. Competence in interaction mechanics is very general, transferring to all contexts within which the same interaction technologies are used; however, the contribution of a particular game to the development of this competence is likely to be very limited, and certainly no greater than other applications using the same interface technology. In fact, a game may be less effective than other applications that are more demanding in terms of knowledge, for example, of keyboard layout, such as word processors. Competence in interaction semantics transfers only to other systems using the same mappings from mechanical interaction operations to in-game actions. This may include many other games, especially those within the same genre but also across genres, depending upon their adoption of implicit or explicit conventions in game-interaction design. However, interaction semantics may be limited in their transferability to other contexts, since contemporary methods of triggering synthetic actions synthesized by a computer game are unlikely to be the same as methods of realizing actions that are not synthesized by a computer.

Game play competence has similar transferability across computer games to competence in interaction semantics, that is, high transferability within a genre but decreasing across genres. However, the potential for transfer of game play competence to contexts other than computer games may be much greater, since similar cognitive processes implementing a sense->model->evaluate->plan->act sequence could apply within those contexts. For example, a flight simulator based upon accurately modeled flight planning and air traffic control procedures may help players to learn how to manage flight planning and air traffic control operations in a real flying context. The key issue here is whether the particular mechanics and design features of the game lead to the development of cognitive structures that can transfer to other contexts. The effectiveness of computer games as situated learning environments (as characterized by [2]) critically depends upon this issue of transfer.

The nature of the cognitive structures underlying game play is not only relevant to knowledge and skill transfer. Those structures are the key to therapeutic applications of game play (e.g., [3]), and in fact are the key to the ability of computer games in all contexts to engage and immerse players and motivate ongoing play. This follows since it is the game play schema driving the situated decision process that determines the nature and timing of emotional rewards motivating play. Hence, a greater understanding of the cognitive structures underlying game play and how motivations and rewards are related to these can aid in better game design in entertainment, pedagogy, and therapy. More than this, it is the central claim in this paper that explicitly modeling those cognitive structures and processes within a computer game engine has the potential to greatly enhance design effectiveness by providing the foundations for the game system itself to guide the development of cognitive structures and control the emotional rewards underlying play.

This paper explores this issue by first considering the cognitive framework for analyzing game play described by Lindley and Sennersten [4]. Methods for conducting analyses of play with a view to identify underlying game play schemas are then described. Based upon this, the paper goes on to consider potential methods by which a computer game system might itself form hypotheses about the schemas underlying the play of a particular game. Finally, we consider some ways in which hypothesized game-play schemas can be used automatically within a computer game system to modify game mechanics as a basis for guiding play and influencing ongoing schema formation and refinement on the part of a player. This work differs significantly from many past projects to create computational players (e.g., see [5]) in that the latter are typically focused on optimal game-play methods that do not need to use computational techniques based upon human play performance. In the case of the work described here, the particular strength and interest of the method are the characterization and explicit representation of the specific algorithmic strategies and cognitive processes of human players, both for analytical purposes and as a foundation for adaptive game mechanics.

\section{A COGNITIVE THEORY OF GAME PLAY: TASKS, ATTENTION, SCHEMAS, AND THE PLEASURES OF PLAY}

Lindley and Sennersten [4] present a theory of the underlying cognitive systems involved in game play based upon schema theory and attention theory. Schemas are cognitive structures that link declarative (or factual) and procedural (or performative) knowledge together in patterns that facilitate comprehension and the manifestation of appropriate actions within a context. While the taxonomical structures 
of semantic or declarative memory are comprised of object classes together with associated features and arranged in subclass/superclass hierarchies, the elements of schemas are associated by observed contiguity, sequencing, and grouping in space and/or time [6]. Schemas can refer to declarative knowledge and taxonomical types with their features and relationships, and integrate these with decision processes. Schemas include scripts for the understanding and enacting of behavioral patterns and routines, a classic example being Schank and Abelson's [7] example of the restaurant script that includes a structure of elements for entering a restaurant, sitting down, ordering food, eating, conversing, paying the bill, leaving, and so on. Scripts, as structures used for both comprehension and behavior generation, represent a structure of cognitive functions that may include cognitive resources, perceptual interpretations and preconditions, decision processes, attention management, and responsive motor actions. Story schemas are patterns representing a structure of understandable elements that must occur to make stories comprehensible. The presence of story schemas in the cognitive systems of storytellers, listeners, readers, or viewers of stories allows stories to be told and to be comprehended, including the inference of missing information. If a story deviates too far from a known schema, it will not be perceived as a coherent story. Script and story schemas are concerned with structures of both space and time, while scenes are schemas representing spatial structures, such as the layout of a house, a picture or an area of a city.

While schemas have been interpreted in many different ways, here a game play schema is understood as a cognitive structure for orchestrating the various cognitive resources required to generate motor outputs of game play in response to the ongoing perception of an unfolding game. A game play schema is therefore the structure and algorithm determining the management of attentional and other cognitive, perceptual, and motor resources required to realize the tasks involved in game play. Examples of types of game-play schemas described by Lindley and Sennersten [4] include story scripts for understanding high level narrative structures designed into games, and scripts for the combative engagement of an enemy, exploring a labyrinth, interacting with a trader nonplayer character, and negotiating and carrying out quests.

Attention theory provides an account of the energetic resources available to cognition, together with principles for the distribution of energy (or attention) to the cognitive resources that use (or manifest) it. Attention theory addresses issues of attentional focus, management of attention (including attentional selection), and the allocation of cognitive resources to cognitive tasks. Ongoing research is addressing the question of the detailed operation of attentional mechanisms, including questions such as the degree to which attentional capacity is specific to specific cognitive resources (or modes) or sharable among resources according to demand, and the stage of processing of perceptual information at which perceptual information is selected for attentional priority. Schemas can be regarded as mechanisms or algorithms that, among other functions, determine the allocation of attention to cognitive tasks.
In the context of game play, attention and the operation of game play schemas are driven by hierarchical goals that set tasks for a player. Goals include those intended by designers and those created by players as allowed by a game design. A hierarchical decomposition of game play goals might at a high level include the completion of a game, which decomposes into the subgoals of finishing each of its levels, each of which in turn decomposes into goals of completing a series of game challenges (and other tasks invented by the player).

We hypothesize that this hierarchical goal structure is mirrored in a hierarchical structure of schemas within a player's cognitive system, where a schema is an algorithm for completing a particular goal or subgoal. As argued by Lindley and Sennersten [4], this schema structure is fundamental to many aspects of the pleasures and motivating factors behind play. These include the pleasures of the following.

(i) Effectance which is a basic feeling of empowerment created when an action of a player results in a response from the game system [8]. The cause-effect relationships underlying effectance are a fundamental premise of goal-oriented schemas for action.

(ii) Closures at different hierarchical levels (as described by Holopainen and Meyers [9]), where a closure is interpreted here as the completion of the algorithm constituted by a play schema. Closures may involve completion of expected outcomes and resolution of dramatic tensions, corresponding to the completion of cycles of suspense and relief identified by Klimmt [8]. A distinction must be made here between the intrinsic pleasures of schema completion and more complex emotional experience and rewards due to fictional identification within the game world (see the point below regarding episodes).

(iii) Achievement of in-game tasks which is rewarding due to the displacement of a player's identity into their character [9], this being a matter of imaginative immersion as described by Ermi and Mäyrä [10]. Achievement-oriented reward is a more specific form of reward than mere closure, since it is associated with the completion of schemas by the achievement of specific goals.

(iv) More complex forms of enjoyment in game tasks regarded as episodes [8] following from imaginative displacement into the game world. Enjoyment within episodes may include the excitement of possible action, the pleasures of curiosity and discovery, the pleasures of experiencing negative emotions of suspense followed by the transference of arousal to an ecstatic experience when the challenge creating the anxiety of suspense is overcome, and enhanced self-esteem. Schemas offer greater discrimination of the pleasures involved in episodes by allowing different forms of episodes to be modeled as different schema patterns having a complex substructure with corresponding emotional effects (e.g., different scripts for solving mysteries, combat, exploration, trading, and quest negotiation). 
(v) Escape to an alternative reality provided by the fictional world represented by a game [8] and facilitated by imaginative displacement. Players have the pleasure of being able to experience new objects, actions, social interactions, and experiences at no risk. These vicarious experiences can help players to cope with felt frustrations and deficiencies in their everyday lives, a process both of catharsis and of perception of increased competence and relevance. Schemas for stories facilitate displacement, while many additional schema forms provide the foundations for comprehension of the events within the fictional world and provide mechanisms for projection of the player's sense of self into the fiction.

(vi) Achievement of a sense of flow [11] in game play, this being a state at the boundaries between engagement and immersion, of being totally absorbed in meeting a constantly unfolding challenge. We hypothesize that the flow state is associated with attentional demand, in particular occurring when schema execution demands attentional resources above a level that would result in player boredom and below a level that would result in excessive difficulty and consequent frustration.

Schema theory therefore has the potential to provide both an explanation of the decision and operational processes underlying game play and an explanation of the detailed reward and motivation factors behind play. Validating this potential requires detailed study of play resulting in the development of empirically validated hypotheses about the detailed structure and functionality of game-play schemas, for individual players and across groups of players.

\section{METHODOLOGIES FOR IDENTIFYING GAME PLAY SCHEMAS}

Identification of game play schemas is a knowledge acquisition and representation process. Our current methodology for doing this includes analysis of the design features of test games, logging of player key strokes and mouse movements, recording of the screen history of play, eyetracking data showing the locus and dynamics of player gaze behavior, and think-aloud protocols to gain some insight into the player's conscious experience of play and its decision processes. Analysis of this data then proceeds by a process of detailed analysis of individual play sessions in order to identify different play modes and abstract hypothetical underlying game play schemas. This in itself is a complex process that may begin with cognitive task analysis (CTA, see http://mentalmodels.mitre.org/cog_eng/ce_methods_I.htm), but must end with a detailed cognitive explanation of the decision processes involved in terms of basic cognitive functions. Statistical patterns of play interaction (mouse moves, key strokes, and eye movements) that may correlate with the presence and execution of specific game play schemas are then identified. This requires the separation of an analysis dataset from which schema models and initial statistical distributions are derived from a test dataset that can then be used to validate those schema models. This sequence is iterated in order to refine the identified schema models.
The design features of the games used within these studies are crucial. Hence, an initial analysis of the selected games must be made in order to identify their general features. The iterative process of refining and validating hypothetical game play schemas must also involve the creation of purposespecific test games or levels, this being done by level editing and modding (i.e., modification of off-the-shelf games, potentially including their media content and scripted behavior). It is also possible to implement a hypothetical schema to create a computational player and to test the resulting game play interactions with actual player interaction as another method of validating a schema hypothesis. As noted by Lindley and Sennersten [4], a CTA provides the first approximation description of a game play schema, but a CTA is also heavily determined by the language and cultural constructs of the observer. The phenomenologically meaningful terms of a CTA may have to be further analyzed to account for the ways that those high-level constructs are actually realized by underlying neurophysiological mechanisms, and this mapping could involve different parsings of functional units at the CTA and neurophysiological levels. Hence, a game play schema might be described at different levels of abstraction or from different interpretation perspectives, some being meaningful in terms of the subjective languages of task performance (e.g., the terms of self-reported task performance) or CTA and others in terms of implementational neurophysiology that may have a very different structure and functional decomposition than that of more linguistically conditioned accounts.

The choice of the level of abstraction in game play schema descriptions may depend upon the purpose of the analysis. More importantly, however, it may be that distinctive statistical profiles can be associated with schema characterizations at an optimal level of abstraction; more abstract schema descriptions may be too general to have any statistical discrimination between them, while more detailed descriptions may involve details that cannot be correlated with statistical groups. Hence, an important ongoing task is the statistical validation of suitable levels of schema description. It is yet to be determined how consistent the level of description needs to be, across game genres, games within genres, different kinds of players, different players within those types, and different play sessions for the same player. It is hoped, however, that applying this methodology will result in statistical profiles uniquely associating player types and game design feature sets with distinctive statistical distributions of interaction primitives at the level of interaction semantics that indicate specific hypothetical game play schemas (or sets of schemas) within the cognitive systems of those players. This is a large undertaking (and in fact endless, as game design continues to evolve) that must be approached incrementally by focusing upon specific genres, games, and design feature subsets.

Questions of levels of abstraction and also of higher-level structures also apply to interaction primitives. Basic interactions implement game moves at the semantic level. However, the presence of specific schemas may be indicated by specific sequences or clusters of interaction semantics, rather than, or in combination with, their frequency. Different play modes, 
such as setting options versus game play commands directed towards achieving in-game goals (i.e., game moves) can often be distinguished by specific discrete interaction primitives, such as hitting an escape key. However, manual interpretation of play and the formation of schema hypotheses based upon this are crucial for defining criteria for distinguishing between the presence of different schemas that involve the same or similar interaction primitives.

To illustrate this discussion, a hypothetical schema can be described based upon observations of play of the role playing game Neverwinter Nights. Neverwinter Nights is a thirdperson point of view game in which the player has a primary in-game character and this character can gain a number of companions in order to form a team, also controlled by the player. Within the game world there are many underground labyrinths consisting of rooms and chambers connected by passages. Rooms and passages often have doors and the labyrinths in general contain threats such as monsters and traps, nonplayer characters that may be friendly or hostile depending upon how the player character interacts with them, powerups and various treasures. The play patterns observed for this example occurred within a period of the game during which the player is intended (by the game designers) to be acting to achieve a number of higher-level story goals predesigned into the game; in particular, the player is on a quest to find four specific creatures that are the key to creat an antidote for a plague and each of which is hidden somewhere within its own labyrinth. Each labyrinth has a similar abstract structure and distribution of game challenges, with differences in its thematic realization. This leads to a style of game play that manifests highly repetitive patterns of interaction and decision making. The schema, expressed in this case in a kind of high-level and informal pseudocode, is a hypothesis about (part of) the underlying algorithm responsible for manifesting these repetitive patterns as the player character and team move through the labyrinths.

The question of the level of abstraction involved is illustrated by considering a significant number of possible subtasks and additional tasks that are not represented in the above description: Check health bar for $1, \ldots, \mathrm{N}$ characters, Check for treasure/items to pick up, Check item attributes/quest relevance, Select navigation waypoints for movement, Avoid enemies during retreat, Tweak group member positions, Bring back strays, Check status of quests, Talk with NPCs, Accept/reject quests, Check minimap window, Reconfigure inventory, Reconfigure equipped items, Select level up options, and so forth.

A complete schema description must include all possible subschemas and include a way of representing the operation of simultaneous parallel schemas, their relative priority, and the principles for switching from one schema to another. The detail involved can be high. For example, the detailed description of a subtask such as "check map window" must include an account of exactly what it is that is being looked for in the map window, how the data is to be interpreted, and some kind of representation for the outcome of the minimap check (e.g., a decision about being lost and/or activating a goal-related reorientation subschema).
1. Stop at Closed Door

2. Check health of party if $>1$ party member low, then: Rest Party Resummon summoned creature else if 1 party member low, then: if lots of healing potions, then: administer healing else Rest Party

3. Enter combat configuration Resummon summoned creature

4. Open door and enter room

5. If there is an enemy

Select target

Monitor health of party until enemy defeated if $>1$ party member has low health, then:

Run away

Rest Party

Resummon summoned creature

Go back to step 3

else

if 1 party member low, then:

if lots of healing potions, then: administer healing

else

Run away

Rest Party

Resummon summoned creature

Go back to step 3

6. If enemy remains, go to step 5

7. Check for traps ...

... etc....

Algorithm 1

\section{AUTOMATED IDENTIFICATION OF GAME PLAY SCHEMAS AND SCHEMA-BASED ADAPTATION OF GAME MECHANICS}

The intended outcome of schema analysis over significant numbers of players and play sessions is a probabilistic profile of the frequencies, clusters, and/or sequences of semantic interaction primitives (game moves) associated with different types of underlying game play schemas for a specific game (i.e., its design features). If such a set of statistical profiles is available, it may be possible to use the profiles for automated identification of the schemas of particular players/play sessions. It is possible to automatically record (or $\log$ ) interaction semantics for a particular player during a particular play session or across different play sessions. This will result in a count of the absolute frequency of each type of semantic interaction primitive used by that player, which can be turned into a relative frequency by subdivision with the total count of interaction primitives. This might be used (in addition to specific commands that indicate changes in play mode) to match against a database of statistical profiles of different game play schemas in order to derive a probabilistic 
hypothesis about the likelihood that specific known schemas are underlying play. Here, we hypothesize that poor overall correlations are likely to indicate the presence of previously uncharacterized schemas, which ideally should be returned to a central schema repository for the game for analysis and new schema description development and distribution.

As described above, game play schemas represent the significant learning outcomes of game play and also encapsulate various rewards of play. An explicit representation of desired and observed game play schemas within a game system constitutes a knowledge base that can potentially be used automatically for a variety of purposes by the game system.

Schema representation and mapping can be used for automated monitoring of a game design as a method of validating the design in terms of player satisfaction. Since a schema includes various points of player reward and presents a time structure for the emotional experience of a game, the schema indicates a hypothesis about the nature of the emotional experience of the player. Different players may prefer different forms of satisfaction. Monitoring schemas and schema execution may indicate which forms of satisfaction particular players are seeking. It may also provide a foundation for determining when a player is not achieving enough satisfaction (based upon criteria that may be derived from a player's history of play, since different players may have different demands in terms of the nature and intensity of rewards). This may be because a game is too easy or too difficult, in which case the game mechanics and parameters determining difficulty for a specific schema can be modified to achieve a better subjective experience. It may also be that a player has not discovered those elements of a schema needed in order for its execution to result in a satisfying experience, in which case the game system model of the player schema related to a target schema might be used to change the game mechanics, for example, to dynamically adapt a level design or to introduce instructional material (perhaps by spawning a suitably informed NPC) to lead the player to actions (such as going to a specific training scenario) that result in gaining the appropriate skills. In effect, this can amount to more efficient and dynamic use of in-game tutorials together with an automation of the normal processes of game tuning carried out manually during game testing prior to release, but having the advantage of being tuned to specific players rather than a group of commercial testers.

Schema descriptions can also be used to explore the effectiveness of a game design in realizing designers' intentions. Simple observations may indicate basic design failures, such as the visual design of interactive elements leading them to be too unobtrusive within the game space for players to notice. However, schema descriptions may show deeper and less obvious problems, such as design features leading too soon to limited modes of play that reward players too much for play patterns that are developed very quickly, discouraging them from exploring a game enough to discover other aspects of its mechanics. As with the other examples presented here, actively using these models within a game system allows the mechanics to be varied for individual players, instead of providing a single solution that is supposed to accommodate everyone.
Monitoring schema formation can also result in automated detection of the degree to which a game design is achieving emergent game play (see [12]) where the design rather loosely constrains the nature of the play experience. In this case, poor correlations with known schemas may be a positive indicator of emergent play. Conversely, design features may be selected that are compatible with a broad set of known schemas representing very different play styles, ensuring that a design accommodates a wide variety of play styles, a space within which players have a lot of freedom to create patterns of interaction.

A major use of explicit schema representations may lay in pedagogical or therapeutic functions of game play. In this case, target schemas may not be initially derived from game play but from the target application domains for learning or training. For example, in a military application, observation of tactical decision making in the field could support the development of schema descriptions for tactical decision making. A game for tactical training should then encourage players to preferentially develop the same or, functionally, similar schemas. The effectiveness of the design of a game intended for tactical training can then be assessed by comparing the schemas of players derived from observation of their play patterns with those of operational tacticians. This may be a great advantage compared to assessing performance outcomes, since performance outcomes alone only indicate how a player has mastered a game system, with no indication of how well the mastery of the game will transfer to an application domain. The schema description is an explicit representation of the cognitive capabilities that facilitate operational competence, thereby having much greater transfer potential from the game to the target application environment. Moreover, aspects of the operational schemas that cannot be facilitated by game design provide an explicit representation of the limits of transfer that may then be used to appropriately focus on supplementary training.

Just as in the case of tuning game mechanics for player satisfaction, explicit schema representations and monitoring of player schemas can be used to adapt game mechanics to achieve pedagogical or therapeutic outcomes. For example, a game designed to train players to achieve batter spatial navigation skills might present an initial diagnostic level involving a comparatively complex navigation task based upon a variety of cues, such as verbal descriptions, minimaps, distance cues, and local cues like footprints and vehicle tracks. Based upon which cues players use, ongoing levels can reduce or exclude cues that are already taken into account and emphasise neglected cues to encourage the development of broader attention patterns.

\section{CONCLUSION}

This paper has described an approach to the analysis of game play based upon schema theory and attention theory. An empirically based method has been described as a basis for identifying and validating hypothetical game play schemas. Automated schema recognition and the potential uses of explicit schema representations within game systems have been explored. This approach provides for explicit modeling of the 
cognitive systems and processes underlying game play, both for analytical studies of play and as a potential implementation mechanism for adaptive games. Work on the analysis of games using this approach is ongoing. It is hoped that the results of this work will provide the foundations for future implementation of schema-based adaptive game systems.

\section{ACKNOWLEDGMENTS}

This paper has been written in the context of the FUGA (FUn of GAming) EU research project, and a collaboration with the Swedish Defence Research Agency (FOI). We thank our colleagues for many stimulating inputs to this project.

\section{REFERENCES}

[1] A. Rollings and E. Adams, Andrew Rollings and Ernest Adams on Game Design, New Riders, Indianapolis, Ind, USA, 2003.

[2] J. P. Gee, What Video Games Have to Teach Us About Learning and Literacy, Palgrave Macmillan, New York, NY, USA, 2003.

[3] G. Robillard, S. Bouchard, T. Fournier, and P. Renaud, "Anxiety and presence during VR immersion: a comparative study of the reactions of phobic and non-phobic participants in therapeutic virtual environments derived from computer games," Cyberpsychology \& Behavior, vol. 6, no. 5, pp. 467-476, 2003.

[4] C. A. Lindley and C. C. Sennersten, "A cognitive framework for the analysis of game play," in Proceedings of the 28th Annual Conference of the Cognitive Science Society: Workshop on the Cognitive Science of Games and Game Play (CogSci '06), Vancouver, Canada, July 2006.

[5] H. J. van den Herik, Y. Björnsson, and N. S. Netanyahu, "Computers and games," in Proceedings of the 4th International Conference (CG'04), Ramat-Gan, Israel, July 2004, Revised Papers. Lecture Notes in Computer Science 3846 Springer 2006.

[6] J. M. Mandler, Stories, Scripts and Scenes: Aspects of Schema Theory, Lawrence Erlbaum Associates, Hillsdale, NJ, USA, 1984.

[7] R. Schank and R. Abelson, Scripts, Plans, Goals and Understanding, Erlbaum, Hillsdale, NJ, USA, 1977.

[8] C. Klimmt, "Dimensions and determinants of the enjoyment of playing digital games: a thrre-level model," in Proceedings of Level Up: Digital Games Research Conference, M. Copier and J. Raessens, Eds., pp. 246-257, Utrecht, The Netherlands, November 2003.

[9] J. Holopainen and S. Meyers, "Neuropsychology and Game Design," Consciousness Reframed III, Newport, Walse, UK, http://www.stephan.com/NeuroBio.html, May, 2006.

[10] L. Ermi and F. Mäyrä, "Fundamental components of the gameplay experience: analysing immersion," in Proceedings of the Digital Games Research Association Conference, Changing Views: Worlds in Play (DIGRA '05), S. de Castell and J. Jenson, Eds., pp. 17-25, Vancouver, BC, Canada, June 2005.

[11] M. Csikszentmihalyi, Flow: The Psychology of Optimal Experience, Harper Perennial, New York, NY, USA, Reproduction edition, 1991.

[12] K. Salen and E. Zimmerman, Rules of Play: Game Design Fundamentals, MIT Press, Cambridge, Mass, USA, 2004. 

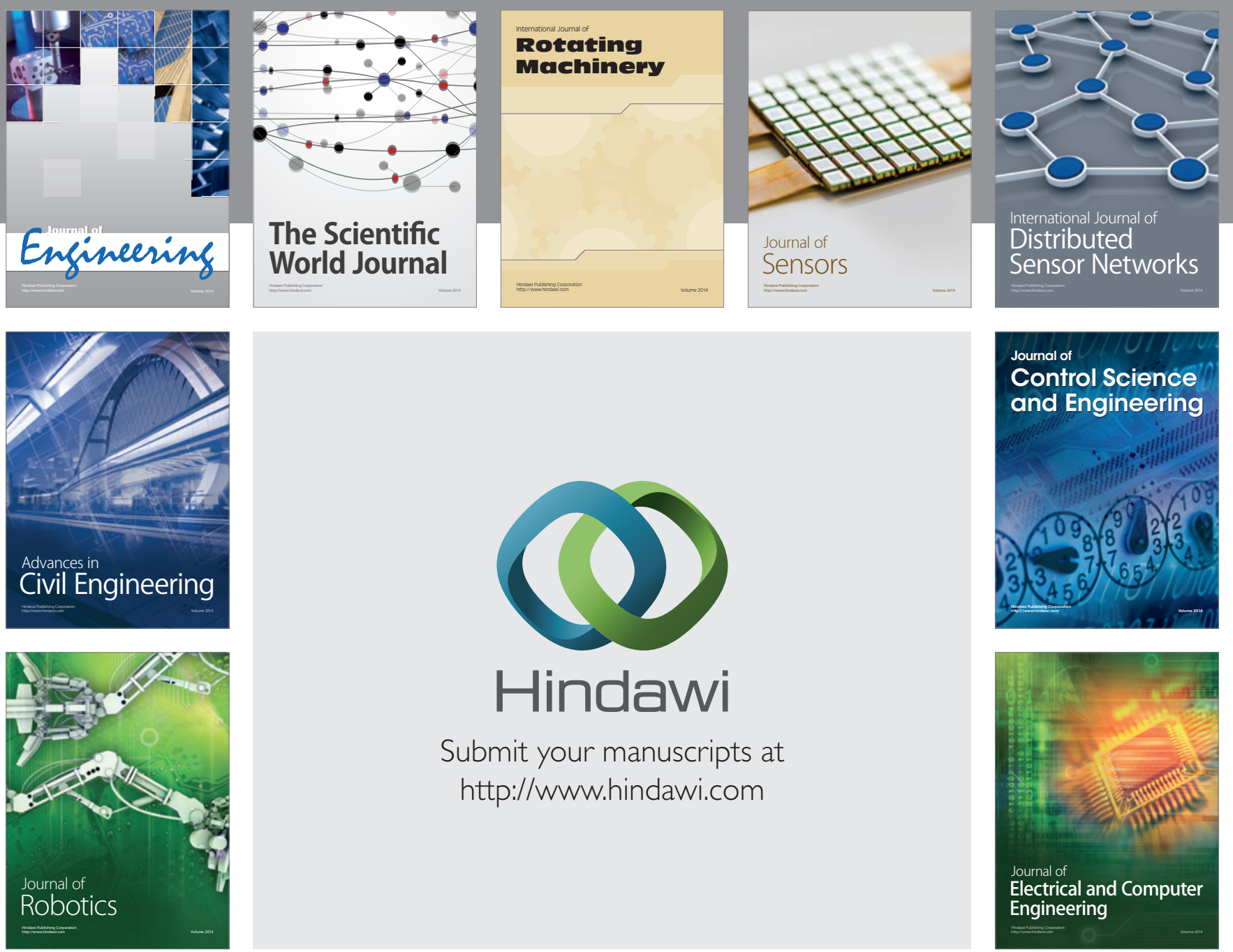

Submit your manuscripts at

http://www.hindawi.com
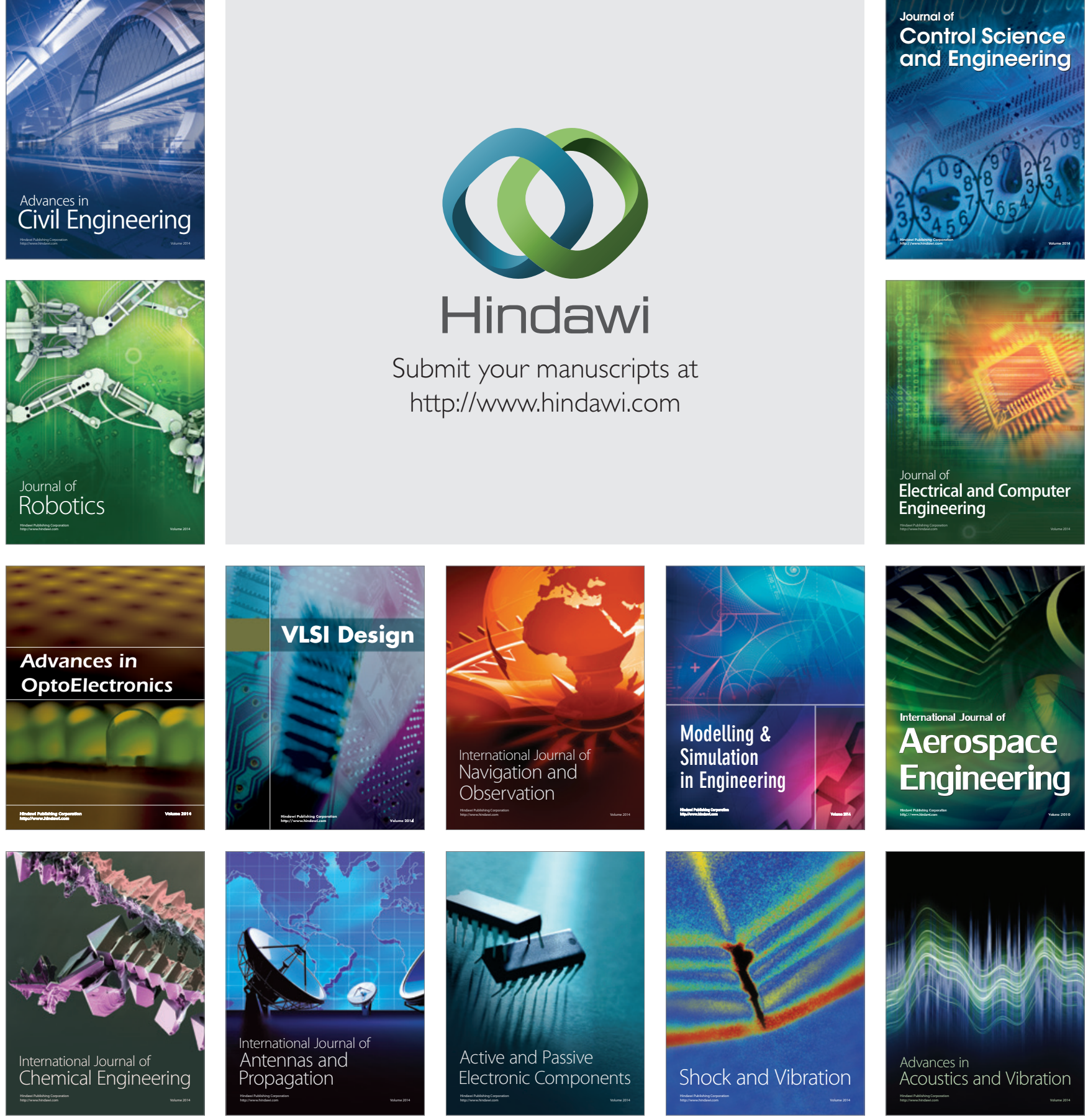